\title{
Computer Simulation on Structural Plane of Ore-rock By Monte-Carlo Method
}

\author{
Pan Dong ${ }^{1,2, a}$, Li XiBing ${ }^{1, b}$, WangYu ${ }^{1, c}$ \\ ${ }^{1}$ Central South University, ${ }^{2}$ Changsha Institute of Mining Research, Changsha Hunan, 410012 China, \\ a pan.dong@163.com, ${ }^{\mathrm{b}}$ xbli@csu.edu.cn, ${ }^{\mathrm{c}}$ 396254855@qq.com
}

Keywords: Monte-Carlo method, structural plane, computer simulation

\begin{abstract}
Based on Monte-Carlo Method, found a computer method for simulating structural plane of ore-rock with partial actual-data. It had proved that the method can reflect the true characteristics and distribution rule of structural plane of ore-rock, thereby provided reliable means of block analysis and forecast.
\end{abstract}

\section{Preface}

It is one of the preconditions for fragmentation prediction that research on structural plane of ore-rock and fixing the features and regularities of distribution of structural plane in the mines which with natural block caving method. Based on the research of drilling and roadway structure surface, the paper determined the dominant group and its occurrence of structural plane, after that, by use of Monte-Carlo method and population distribution on simulation of structure plane, provide reliable basis for group characteristics and spatial distribution pattern of structural plane of lean ore and migmatite and further rock fragmentation analysis forecast.

\section{Basic principle of computer simulation for structure plane}

\section{Basic principle of stochastic simulation by Monte-Carlo method}

It is proved that occurrence factors in structure plane, just as inclination, tendency, trace length and spacing of structural plane have good geometry or probability distribution law. Computer simulates the actual distribution of structure plane by using measured occurrence factors and determined distribution and parameters. This process is contrary to the structural plane occurrence determined in the field work. Field test statistics is based on the ore and rock structural plane distribution form, then get the characterization of structural plane distribution features of various geometric parameters, determine the probability distribution function and the distribution parameters. And Computer simulation of rock structural plane is the reverse process, just According to the structure surface of measured data, determine the occurrence factors, probability distribution function, establishing the distribution probability model, simulate and model established by the similar or parallel random number, through the sample statistical sampling, calculating distribution models for the estimation of parameters, thus obtained by computer simulation of structure plane distribution model of geometric figure, so as to realize the computer simulation of the structure faces.

\section{Probability Generating Function Method}

Monte-Carlo method is based on a certain distribution random sampling numerical average score. By means of computer simulation, the first task is how to generate random numbers. Because of the real random number in the computer to complete, and in the numerical simulation for numerical tests are reproducible, and thus in the simulation of actual use is called "pseudo random number". In rock discontinuity network simulation, the key is how to generate $[a, b]$ interval distribution (denoted as $u$ $[a, b])$ "pseudo random number". Random number generation mathematical methods usually include middle-square method, shift instruction addition and congruence method. Congruence method includes multiplicative congruence method, additive congruence method and mixed congruence method three. They are selected according to the initial value, determined by an iterative process to achieve. At present the most commonly used is a multiplicative congruence method, has been widely used. This study used the congruence pseudo-random number generation. 


\section{Distribution types of structural plane parameters}

Structural plane main parameters of rock mass include inclination, tendency, trace length, space composition, and the parameter values are generated by a corresponding probability function. In order to simulate structure model of rock mass, we must first determine the probability function model and parameters of geometric parameters of structure plane. There are many scholars domestic and overseas have researched on structural plane spacing, trace length distribution function form and space shape assumption.

Based on field investigation and statistics of structural plane of the borehole and drift analysis, this paper concludes the probability distribution types and parameters of various geometric parameters of structural plane. The structure surface tendency and inclination distribution of mixed rock and lean ore are shown in table 1, its confidence deviation values are shown in table 2.

Table 1 Preferred structural plane occurrence features of mixed rock and lean ore

\begin{tabular}{|c|c|c|c|c|c|c|c|c|c|c|}
\hline \multirow{2}{*}{ Lithology } & Distribution & Parameter & \multicolumn{3}{|c|}{1} & \multicolumn{2}{|c|}{2} & \multicolumn{2}{|c|}{3} & \multicolumn{2}{|c|}{4} \\
\cline { 3 - 12 } & form & statistics & Mean & Variance & Mean & Variance & Mean & Variance & Mean & Variance \\
\hline \multirow{2}{*}{$\begin{array}{c}\text { Mixed } \\
\text { rock }\end{array}$} & Normal & Tendency & 193 & 28 & 287 & 78 & 354 & 26 & 91 & 22 \\
\cline { 3 - 13 } Lean ore & $\begin{array}{c}\text { Normalion } \\
\text { distribution }\end{array}$ & Inclination & 67 & 10 & 84 & 18 & 62 & 12 & 31 & 48 \\
\cline { 3 - 12 } & Tendency & 87 & 43 & 136 & 33 & 324 & 21 & 182 & 35 \\
\hline
\end{tabular}

Table 2 Confidence deviation values of inclination and tendency of mixed rock and lean ore

\begin{tabular}{|c|c|c|c|c|c|c|}
\hline Lithology & confid & tural plane & 1 & 2 & 3 & 4 \\
\hline \multirow{4}{*}{ Mixed rock } & \multirow{2}{*}{$90 \%$} & Inclination & 1.257963 & 2.862507 & 1.935666 & 7.818205 \\
\hline & & Tendency & 3.522296 & 12.4042 & 4.193944 & 3.583344 \\
\hline & \multirow{2}{*}{$95 \%$} & Inclination & 1.498849 & 3.410646 & 2.306326 & 9.315308 \\
\hline & & Tendency & 4.196778 & 14.77947 & 4.997039 & 4.269516 \\
\hline \multirow{4}{*}{ Lean ore } & \multirow{2}{*}{$90 \%$} & Inclination & 2.030632 & 2.730762 & 4.935 & 3.455985 \\
\hline & & Tendency & 10.91645 & 4.742902 & 5.7575 & 7.559968 \\
\hline & \multirow{2}{*}{$95 \%$} & Inclination & 2.419477 & 3.253674 & 5.88 & 4.11777 \\
\hline & & Tendency & 13.00469 & 5.651118 & 6.86 & 9.007621 \\
\hline
\end{tabular}

According to the formula 1, trace length is,

$l=\frac{n_{1}+2 n_{0}}{2 N} \frac{\pi \cdot w \cdot h}{w+h}$

In the formula,

$n_{1}$-The number of structure plane that One of end is visible;

$n_{0}$ - The number of structure plane that both end are invisible;

$N$-The total number of structural plane, $N=n_{1}+n_{2}+n_{0}\left(\mathrm{n}_{2}\right.$ is the number of structure plane that both end are visible).

Calculate, trace length of rock structural plane are shown in table 3.

Table 3 Estimated results of mean trace length $(\mathrm{m})$

\begin{tabular}{|c|c|c|c|c|c|}
\hline Lithology & $\begin{array}{c}\text { Distribution } \\
\text { form }\end{array}$ & 1 & 2 & 3 & 4 \\
\hline Mixed rock & $\begin{array}{c}\text { Negative } \\
\text { exponential }\end{array}$ & 1.5047 & 0.6664 & 1.1923 & 0.6686 \\
\hline Lean ore & $\begin{array}{c}\text { Negative } \\
\text { exponential }\end{array}$ & 3.9130 & 1.7534 & 0.5271 & 1.1985 \\
\hline
\end{tabular}

Data of average spacing obtained from actual measurement are shown in table 4 .

Table 4 Mean spacing of structural plane of mixed rock and lean ore $(\mathrm{m})$

\begin{tabular}{|c|c|c|c|c|c|}
\hline Lithology & $\begin{array}{c}\text { Distribution } \\
\text { form }\end{array}$ & 1 & 2 & 3 & 4 \\
\hline Mixed rock & $\begin{array}{c}\text { Negative } \\
\text { exponential }\end{array}$ & 0.15496 & 0.2727 & 0.0873 & 0.5049 \\
\hline Lean ore & $\begin{array}{c}\text { Negative } \\
\text { exponential }\end{array}$ & 0.0891 & 0.2389 & 0.2536 & 0.2402 \\
\hline
\end{tabular}


According to the joint spacing negative exponential fitting formula $y=A \cdot e^{-B x}$, performed the regression analysis to the rock group, the results are shown in table 5.

Table 5 Parameters(A, B) value of histogram fitting formula of various lithology joint spacing

\begin{tabular}{|c|c|c|}
\hline \multirow{2}{*}{ Lithology } & \multicolumn{2}{|c|}{ Parameters value of fitting formula } \\
\cline { 2 - 3 } & $A$ & $B$ \\
\hline Mixed rock & 0.1658031435 & 1.856094893 \\
\hline Lean ore & 0.06165329309 & 1.377900938 \\
\hline
\end{tabular}

\section{Design of computer simulation program of structural plane}

\section{Basic assumptions of network simulation of structural plane}

The computer graphics simulation of structural surface, display as the following agreement,

1) Structural plane occurrence: Inclinations, tendencies obey normal distribution, and spacing, trace lengths obey negative exponential distribution.

2) Structural plane surface is a circular disc shape.

3) Starting point and terminal point coordinate the uniform distribution of subject area.

4) In the simulation, linear representation structure plane traces, trace occurrences are determined by $\alpha$ which azimuth of straight line is. $\alpha$ is the angle between $\mathrm{X}$-axis and trace(counterclockwise is positive direction). Structural plane occurrence and simulated map section direction determine the size of trace azimuth angle $\alpha$.

\section{Program of structural plane simulation}

The steps of computer simulation of rock structure plane are following:

1) Calculation parameters input. It is including: simulation area scope definition, simulation profile azimuth, statistical parameters of structural plane characteristics and structural plane group number.

2) Generation of structure plane trace.

a) Structural plane trace midpoint is uniform distribution, then the starting points coordinates (xoi, yoi) of structure plane trace are determined by means of sampling of Monte-Carlo method.

b) According to the probability distribution function of tendency, inclination and trace length, the specific numerical of these parameters can be determined by sampling of Monte-Carlo method.

c) Through conversion, the simulation results of tendency $\beta_{i}$ and projection of trace length $l_{i}$ can be determined by structural plane inclination, tendency and tendency of cutting section simulation.

$\operatorname{tg} \beta_{i}=\operatorname{tg} \beta \cdot \cos \left(\alpha-\alpha_{f}\right)$

In the formula,

$\alpha_{f}, \beta$-tendency and true inclination of the profiles;

$\alpha$-true tendency of the structural plane.

$l_{i}=\frac{l \sin \beta}{\cos \beta_{i}}$

In the formula:

$l$ - trace length of structural plane;

$l_{i}$ - trace length of structural plane profile in the projection plane;

$\beta$-inclination of structural plane;

$\beta_{l}$-inclination of structural plane profile in the projection plane.

d) The parameter which include $x_{\mathrm{oi}}, \mathrm{y}_{\mathrm{oi}}, \beta_{\mathrm{i}}, 1_{\mathrm{i}}$ generate the structure surface in the simulation region, and save them.

3)Repeat second step, until all the trace of structure plane in group are generated.

4) Repeats the above 1)-3) steps for additional structural plane group, and finish all the structure plane computer simulation.

The structure simulation program has the following functions:

1) Realizes the computer visual simulation of structural plane distribution, and regional simulation can be dynamically regulated. 
2) Generates a plurality of sets of structural plane of rock mass.

3) Shows structural plane simulation distribution in different range profile.

4) The results can be saved by common picture format.

\section{The results of computer simulation of ore and rock structural plane}

Through surveying the rock structural plane and analyzing results, the number of structural plane group of mixed rock and lean ore is 4 . According to the arrangement of perforating veins and along veins, Select two profiles which trend are $55^{\circ}$ and $145^{\circ}$ to simulate of structure network. As a result of the stope is placed between a spacing of $12 \times 12 \mathrm{~m}$, the range of structural plane simulation diagram are shown in figure 1 to figure 4.

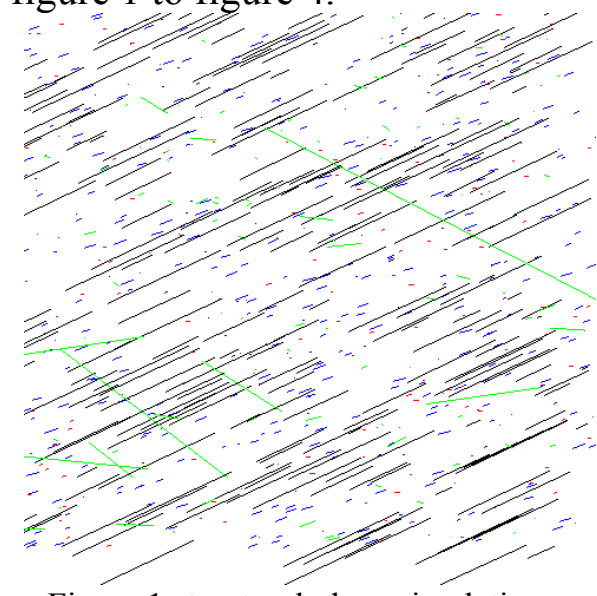

Figure 1 structural plane simulation diagram of $55^{\circ}$ direction and $12 \times 12 \mathrm{~m}$ of mixed rock profile

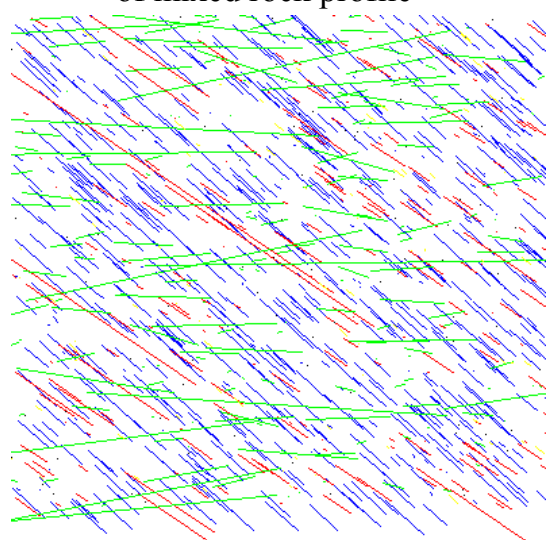

Figure 3 structural plane simulation diagram of $55^{\circ}$ direction and $12 \times 12 \mathrm{~m}$ of lean ore profile

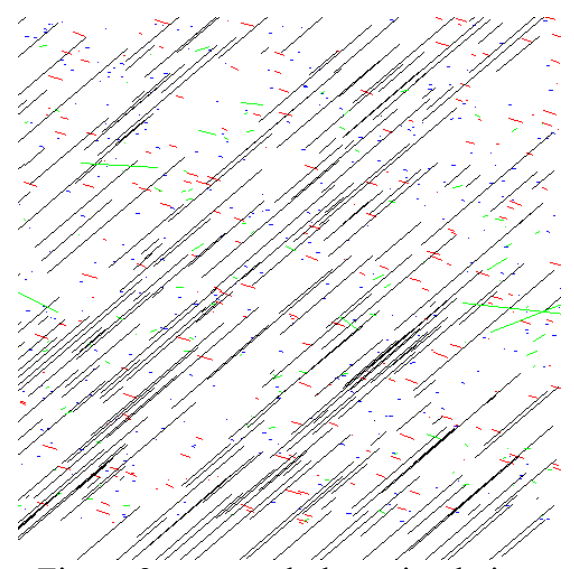

Figure 2 structural plane simulation diagram of $145^{\circ}$ direction and $12 \times 12 \mathrm{~m}$ of mixed rock profile

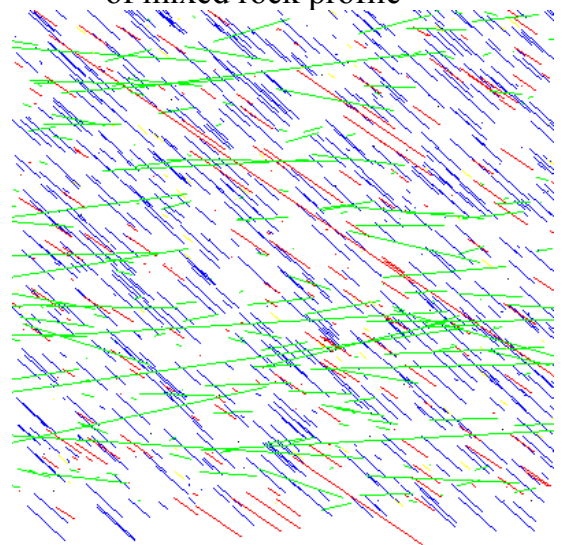

Figure 4 structural plane simulation diagram of $145^{\circ}$ direction and $12 \times 12 \mathrm{~m}$ of lean ore profile

According to the need of analysis, program can generate structural plane simulation diagram in different range and direction of profile.

\section{Conclusion}

Computer simulation of structure network is a kind of advanced research methods, it is proved that the method can actually simulate structural plane of rock mass in the required range, and the result is also very intuitive. According to the simulation results, can further analysis structural connectivity rate, strength parameter of rock mass and some other indexes. Thus in natural caving method, provide reliable basis for the comprehensive evaluation of structural plane distribution and further rock fragmentation analysis forecast.

\section{References}


[1] Jia Hongbiao, Tang Huiming, Liu Yourong, etc. 3D network simulation theory and engineering application of structural plane of rock mass. Science Press. 2008.

[2] Yin Yanbo, Li Aibing, Yuan Jieping, etc. Rock mass structure 2D network simulation computer aided technology research. Mining Technology, 2006, 12.

[3] Pan Wei, Liu Daan, Zhong Huiya, etc. 3D geological modeling and its application to slope engineering. Chinese Journal of Rock Mechanics and Engineering. 2004.

[4] Zhou Tie, Xu Shufang, Zhang Pingwen, etc. Calculation method. Tsinghua University Press. 2006. 